\title{
Mapas personales y cartografía escolar: capacidades del alumnado de primer ciclo de educación primaria ${ }^{1}$
}

\section{Personal maps and school mapping: abilities of students in the first stage of primary education}

\author{
María Jerez Carañana ${ }^{2}$ \\ mariajerez92@hotmail.com \\ Antonio José Morales Hernández \\ Antonio.J.Morales@uv.es \\ Universidad de Valencia, España
}

\begin{abstract}
Resumen:
¿Qué capacidades cartográficas posee el alumnado de primer ciclo de Educación Primaria si se tienen en cuenta sus experiencias personales? Para responder a esta cuestión se ha realizado un estudio de casos en $2^{\circ}$ curso de Educación Primaria, donde se comparan las habilidades cartográficas de un grupo experimental con otro de control. A la muestra se le aplica un pretest y un postest para que realice una representación pictórica de un itinerario y responda a cuatro cuestiones. Los resultados obtenidos se han categorizado según las clasificaciones propuestas por Lynch (1960), Boardman (1986) y Bale (1989) para el análisis del espacio geográfico. Las conclusiones de este estudio de casos muestran indicios que permiten impugnar las capacidades cartográficas
\end{abstract}

\begin{abstract}
:
What cartographic skills do students in the first stage of primary education have taking into account their personal experiences? This question was explored through a case study conducted with Primary Education students in Year 2 in which the cartographic skills of an experimental group were compared with those of a controlled group. The sample was analyzed by means of a pretest and a post-test in which students had to complete a pictorial representation of an itinerary and respond to four questions. The results obtained were categorized according to the classifications for geographical space analysis suggested by Lynch (1960), Boardman (1986) and Bale (1989). The findings of this case study provide indications that challenge the cartographic abilities that evolutionary psycho-
\end{abstract}

1 Este artículo se inscribe dentro del proyecto: «Las representaciones sociales de los contenidos escolares en el desarrollo de las competencias docentes», Proyectos I+D de Generación de Conocimiento y Fortalecimiento Científico y Tecnológico del Sistema I+D+I (Ministerio de Ciencia, Innovación y Universidades), con referencia PGC2018-094491-B-C32, cofinanciado con fondos de la UE.

2 Dirección para correspondencia (correspondence address):

María Jérez Carañana. Dirección: Universidad de Valencia (España). 
Mapas personales y cartografía escolar: capacidades del alumnado de primer ciclo de educación primaria

María Jerez Carañana y Antonio José Morales Hernández

que la psicología evolutiva atribuye a los niños/as de 7 y 8 años, y revelan la importancia de tener en cuenta el espacio vivido para el aprendizaje de la cartografía escolar.

\section{Palabras clave:}

Mapas personales; cartografía escolar; capacidades del alumnado; primer ciclo; educación primaria. logy attributes to the 7-to-8-year-old age range and reveal the importance of taking into account lived space when it comes to learning school mapping.

\section{Key words:}

Personal maps; school mapping; abilities of students; fist stage; primary education.

\section{Résumé:}

Quelles sont les capacités cartographiques des élèves du primaire si leurs expériences personnelles sont prises en compte? Pour répondre à cette question, une étude de cas a été réalisée en 2 e année du primaire, où les compétences cartographiques d'un groupe expérimental sont comparées à celles d'un groupe témoin. Un pré-test et un posttest sont appliqués à l'échantillon afin qu'il puisse faire une représentation picturale d'un itinéraire et répondre à quatre questions. Les résultats obtenus ont été classés selon les classifications proposées par Lynch (1960), Boardman (1986) et Bale (1989) pour I'analyse de l'espace géographique. Les conclusions de cette étude de cas montrent des preuves qui nous permettent de remettre en question les capacités cartographiques que la psychologie évolutionniste attribue aux enfants de 7 et 8 ans, et révèlent l'importance de prendre en compte l'espace vécu pour l'apprentissage de la cartographie scolaire.

\section{Mots clés:}

Cartes personnelles; cartographie scolaire; compétences des élèves; premier cycle; enseignement primaire.

Fecha de recepción: 20-01-2020

Fecha de aceptación: 05-10-2020

\section{Introducción}

La enseñanza de la cartografía escolar queda determinada por los postulados de la Psicología evolutiva (Piaget e Inhelder, 1947; Hanounn, 1977) al asociar los aprendizajes del alumnado con la edad; pero esto puede cuestionarse si, tal y como evidencian otros autores (Thursthone, 1938; Vygotsky, 1962), se tienen en cuenta sus experiencias vividas. Este aspecto condujo a la reflexión de las relaciones existentes entre el espacio subjetivo y absoluto, y animó el estudio sobre el proceso de adquisición de la competencia cartográfica.

Los antecedentes de esta investigación se contextualizan en un Trabajo de Final de Grado (TFG) sobre la construcción del espacio geográfico a partir del mundo subjetivo del alumnado y su posterior desarrollo en la publicación de un artículo en la revista Aula de Innovación Educativa 
(Jerez, 2016) que sirvió para validar la prueba piloto utilizada de referencia en este estudio. Esto permitió plantear, en el marco del Máster de Investigación Didáctica de Ciencias Sociales: Geografía (Facultat de Magisteri, Universitat de València), un Trabajo Final de Máster (TFM) con la finalidad de seguir indagando en las capacidades geográficas de los niños/as de primer ciclo de Educación Primaria (EP), y todo ello centrado en el aprendizaje de la cartografía.

Concretamente se ha realizado un estudio de casos donde hay un grupo control y otro experimental, con el objetivo de evidenciar un mayor desarrollo de las competencias cartográficas en el grupo experimental tras la intervención educativa. La experiencia se ha realizado en segundo curso de EP y desde esta investigación también se pretende impugnar el modelo ofrecido desde la Psicología evolutiva en cuanto a las capacidades cartográficas que tienen los niños/as de 7 y 8 años. Es decir, se pretende demostrar que las destrezas cartográficas no se van adquiriendo en función de la edad, sino que la experiencia y la instrucción previa y progresiva en el conocimiento geográfico es esencial para que esas destrezas se desarrollen antes de lo establecido por la perspectiva piagetiana.

Para entender las dificultades enunciadas es preciso recurrir a la Psicología del Aprendizaje. Según Piaget e Inhelder (1969), la muestra se encontraría en la etapa del pensamiento operacional, donde el niño/a ya empieza a razonar lógicamente sobre los objetos o hechos que observa a su alrededor, aunque las operaciones que realiza sólo podrían aplicarse a objetos presentes. Sin embargo, cuando se trabaja con alumnado de esta edad resulta de interés partir de espacios conocidos para los niños/ as y tener en cuenta que el proceso de enseñanza y aprendizaje debe ser gradual. Desde este posicionamiento pedagógico del conocimiento del espacio se plantea la necesidad de evidenciar si las experiencias vividas por el alumnado influyen en sus habilidades cartográficas.

\section{Planteamiento del problema: hipótesis y objetivos}

Tras lo expuesto se deduce que, según Piaget e Inhelder (1969), el alumnado de segundo curso todavía no estaría preparado para leer o comprender mapas por cuestiones madurativas, ya que el mapa se representa desde una posición vertical -desde arriba-, un punto de vista que dista 
de la visión habitual del niño/a y que le resultará difícil de comprender debido a su egocentrismo y a la baja capacidad de abstracción. Sin embargo, cabe decir que Piaget únicamente hace referencia al espacio concebido, sin tener en cuenta que previamente puede haber un trabajo geográfico desde el espacio vivido o percibido. Hannoun (1977) diferencia estos espacios en tres etapas evolutivas -en la línea de Piaget- para la percepción del espacio por parte del niño/a:

- La etapa de lo vivido. El infante todavía no es capaz de medir distancias, pero sí de vivirlas a través de su experiencia y hacer recorridos mediante el movimiento. Por tanto, el espacio vivido se basa en el contacto directo con un espacio cercano, físico y concreto. Cavalcanti (2017) considera este espacio como el más idóneo para empezar a trabajar el espacio geográfico con los niños/as, pues está compuesto por sus experiencias personales, sus desplazamientos en un lugar, el contexto en el que se desarrollan y los aprendizajes adquiridos, lo cual influye en la percepción.

- La etapa de lo percibido. Ya no se requiere de un contacto directo, el movimiento deja de ser imprescindible porque ahora el espacio se percibe a través de la observación. Aquí se puede trabajar en la percepción de espacios más alejados al niño/a; por ejemplo, se pueden empezar a interpretar fotografías aéreas de lugares conocidos.

- La etapa de lo concebido. Es la visión más externa y objetiva del espacio geográfico, donde el niño/a es capaz de realizar abstracciones y establecer relaciones entre las formas, por ello también se entiende como el espacio matemático. Según el autor esta fase se empieza a alcanzar sobre los 11 o 12 años de edad.

A pesar de que Hannoun y Piaget nos aportan información relevante sobre la forma en la que el niño/a va comprendiendo el espacio, no podemos entender que estas etapas se van alcanzando exclusivamente por la edad del sujeto, sino que es el resultado de un conjunto de factores. Asimismo, Bale (1989) resalta la importancia de indagar en la visión subjetiva que tiene el niño sobre su entorno para poder establecer después una secuencia lógica de actividades; por ello, se partirá de las geografías personales del alumnado para saber cómo percibe y representa el espacio. Según Piñeiro (1998) "entre la realidad y el sujeto se impone un filtro de su personalidad, de sus experiencias, gratas o desagradables, el 
de sus conocimientos y aficiones, el de su cultura. La imagen resultante es la de su percepción" (p.6). Desde esta perspectiva, el entorno queda deformado por las experiencias ambientales y personales, que influyen tanto en la percepción como en el comportamiento geográfico, el cual tiene que ver con el desplazamiento y con la forma de recibir o emitir información espacial (Boira, Reques y Souto, 1994). Tal y como se observa, esta investigación recibe grandes influencias de la Geografía de la Percepción y del Comportamiento, así como de la Geografía Humanista.

Por otro lado, Souto (1998) concreta varios niveles en los que se puede analizar el espacio geográfico y su didáctica con niños/as de entre 3 y 8 años:

- Espacio subjetivo. Es "aquella esfera de comportamiento y actitud que está condicionada por los filtros culturales y emocionales que determinan una valoración de un lugar" (Souto, 2011, p.148). Podría decirse que este espacio se encuentra presente en las etapas de lo vivido y lo percibido, enunciadas por Hannoun (1977).

- Espacio absoluto. Aquel espacio más objetivo, más alejado del punto de vista personal y que está relacionado con lo medible, geométrico, topológico y cartográfico. Este espacio es el que predomina en la didáctica de la Geografía, debido a la concepción tradicional de la misma; y podríamos relacionarlo con la etapa de lo concebido.

- Espacio complejo. Interacción entre el espacio subjetivo y objetivo.

Con todo lo planteado hasta este momento, se entiende que a la hora de trabajar el espacio geográfico con el alumnado es preciso partir de lo más cercano a la realidad de niño y tener en cuenta su visión particular y subjetiva, para después ir extrapolando los conocimientos progresivamente hacia espacios más alejados y con una visión más objetiva. Dicha reflexión permite establecer el objeto de estudio de esta investigación: el aprendizaje cartográfico; y todo ello conduce a la principal pregunta de investigación: ¿Qué capacidades cartográficas posee el alumnado de primer ciclo de Educación Primaria si se tienen en cuenta sus experiencias personales?

A partir de aquí se propone la siguiente hipótesis: el alumnado de primer ciclo de Educación Primaria muestra mayores capacidades cartográficas de las que le asigna la psicología evolutiva si se parte de sus car- 
Mapas personales y cartografía escolar: capacidades del alumnado de primer ciclo de educación primaria

María Jerez Carañana y Antonio José Morales Hernández

tografías personales tal y como evidencia este estudio de casos. También se formulan los siguientes objetivos:

- Indagar desde la literatura científica en las capacidades cartográficas que poseen los niños/as de 7 y 8 años.

- Realizar una diagnosis de la cartografía personal del alumnado de segundo curso de Educación Primaria.

\section{Marco teórico}

Desde una visión tradicional, la Geografía se asocia con aquello que permite organizar el espacio y describirlo, desde un punto de vista objetivo y externo. Sin embargo, en la mitad del siglo pasado adquiere mayor importancia la Geografía de la Percepción y del Comportamiento, donde autores como Lynch (1960) exponen que la visión del espacio no es puramente objetiva ya que cada persona percibe su entorno subjetivamente, de manera que los recuerdos, gustos o experiencias influyen en la percepción y en el comportamiento del observador. Esta corriente se complementa con la Geografía Humanista, pues está centrada en el conocimiento del espacio mediante la vida cotidiana y la propia experiencia. Por este motivo, en la presente investigación, se indaga en la cartografía personal del alumnado desde el inicio para abordar el conocimiento del espacio geográfico desde la vertiente subjetiva.

Para realizar un diagnóstico de las cartografías personales de los niños/as es preciso recurrir a la llamada geografía de la infancia. Este tipo de geografía tiene su objeto de estudio en la vida diaria de los niños/as dentro de su entorno, en cómo crean su identidad y en cuáles son sus espacios deseados (Ortiz, Prats y Baylina, 2011). Una de sus principales técnicas cualitativas es el dibujo y, por ello, se han analizado producciones pictóricas del alumnado sobre un recorrido determinado. De esta manera, los niños/as representan su visión particular del espacio geográfico y la plasman en el papel, lo que da lugar a la elaboración de un mapa mental o cognitivo (Boardman, 1986).

Los mapas mentales están condicionados por la conducta espacial del niño/a en su vida diaria, así como por las experiencias personales y ambientales (Bale, 1989), por lo que a medida que el niño/a va teniendo más experiencias y autonomía espacial irá ampliando esa imagen 
mental del espacio geográfico (Piñeiro, 1998). De hecho, Tonda (2001) establece que los niños de Educación Infantil suelen elaborar mapas mentales basados en unos pocos lugares de referencia o mojones, como la escuela o la propia casa; a los 7 años, los niños/as serían capaces de establecer relaciones entre esos lugares mediante itinerarios o rutas; y a los 9 años los niños/as ya empezarían a conectar varios itinerarios dando lugar a las configuraciones- donde el espacio geográfico queda representado de forma más compleja.

Este tipo de producciones, suelen mostrar una realidad distorsionada por el observador, pues influyen aspectos personales y se emplea una geometría subjetiva, donde la dirección o la distancia no es proporcional a la realidad; mientras que la escala debe ser interpretada y trabajada en las primeras etapas a través del tamaño de los elementos dibujados (Piñeiro, 1998; Marrón, 2012).

A pesar de ello, los mapas mentales son muy útiles porque marcan el punto de inicio para trabajar habilidades cartográficas (Boardman, 1986) y muestran la capacidad que poseen los niños/as para estructurar el espacio, así como para abstraerlo y representarlo. Mientras, el alumnado podrá reflexionar sobre su entorno y sentirse más implicado en la construcción del conocimiento (Piñeiro, 1998).

Llegados a este punto es preciso comprobar cómo se plantea la cartografía escolar desde el currículo, ya que su análisis permite comprender cómo se aborda la didáctica de la Geografía dentro del área de las Ciencias Sociales. En el Decreto 108/2014 de 4 de julio del Consell, por el que se establece el currículo y desarrolla la ordenación general de la Educación Primaria en la Comunitat Valenciana, se expone que la Geografía permite al niño/a desarrollar la capacidad para representar el espacio geográfico mediante planos o mapas, lo que facilitará la reflexión sobre las problemáticas existentes.

Por otro lado, las habilidades cartográficas planteadas desde el currículo se incluyen en el Bloque 2, titulado "El mundo en que vivimos". Cuando se observan los contenidos y criterios de evaluación establecidos, se comprueba que en el primer ciclo de EP sí se propone identificar elementos o lugares de espacios conocidos en planos, así como elaborar croquis a partir de su recuerdo y percepción. Esto último es lo que anteriormente se ha definido como mapa mental y, por lo tanto, el espacio vivido o percibido del alumnado es tenido en cuenta en el primer ciclo de EP y sirve de base para el aprendizaje cartográfico. Sin embargo, 
Mapas personales y cartografía escolar: capacidades del alumnado de primer ciclo de educación primaria

María Jerez Carañana y Antonio José Morales Hernández

la representación espacial no se retoma hasta la Educación Secundaria Obligatoria debido a que el currículo escolar se rige por la psicología evolutiva y se entiende que las habilidades cartográficas se irán adquiriendo por madurez. En consecuencia, los libros de texto presentan escasez de actividades para elaborar mapas y planos, evidenciándose poca diversidad en las mismas (Martínez y López, 2015).

A pesar de todo esto, autores destacados en la didáctica geográfica anglosajona como Boardman (1986) o Bale (1989) argumentan que los niños/as de primer ciclo de EP ya pueden iniciarse en el desarrollo de habilidades cartográficas. Según los autores, la práctica, los mapas mentales y el trabajo sobre la geografía local son elementos importantes para que los niños/as comprendan mejor su entorno y estén más capacitados para integrar elementos cartográficos en planos de espacios conocidos. Por todo ello, la investigación realizada pretende impugnar el modelo de enseñanza de la cartografía expuesto en el currículo escolar, que determina la edad como el principal factor de aprendizaje y desarrollo de las habilidades cartográficas.

De esta forma, se ha diseñado una secuencia de actividades en la que se ha tenido en cuenta aspectos que deben ser básicos en la enseñanza y aprendizaje de los conocimientos científicos, como la organización y una estructura clara de las actividades, la progresión en la dificultad de las mismas y el enfoque constructivista, entre otros (Jiménez, Martínez, Castillo y López, 2019). La finalidad de la secuencia de actividades es que el alumnado adquiera dichas habilidades a través de dos factores clave: la práctica y la experiencia. Para Comes (1993) las habilidades cartográficas pueden definirse como aquellas que permiten al alumnado "saber resolver cualquier problema relativo a la obtención, tratamiento y comunicación de la información que se haga mediante cualquier tipo de documento cartográfico" (p. 28). Por lo tanto en la metodología empleada predomina un enfoque procedimental, es decir, se requiere de la comprensión de los elementos cartográficos para llegar a proceder con ellos.

El desarrollo de la competencia cartográfica se alcanzará una vez adquiridas las habilidades necesarias para interpretar y representar la realidad a través del mapa. Esto permite un aprendizaje integral, ya que no sólo ofrece información de cómo se organiza la sociedad sino también de cómo se representa ese espacio y cómo se va transformando (Francischett, 2016). Asimismo, la adquisición del código cartográfico (formado 
por signos y símbolos) y su interpretación, no es una tarea sencilla para los niños/as lo que contribuirá al desarrollo de las inteligencias múltiples establecidas por Gardner (2003).

Una vez que los niños/as adquieran el código de los mapas, podremos decir que se están iniciando en la alfabetización cartográfica. Dicho proceso no sólo consta de lectura de mapas, sino que también implica que el niño/a conozca su realidad, que sea capaz de representarla y de adquirir una mirada crítica. Con ello, el niño/a podrá detectar problemáticas, reflexionar sobre ellas, plantear soluciones y comunicarlas (Castrogiovanni y Abreu, 2016). Esta idea está relaciona con la Geografía Radical, pues el espacio deja de considerarse como algo estático y pasa a ser entendido como un producto social, donde las personas son las que lo van transformando y organizando (Pillet, 2004).

En definitiva, la alfabetización cartográfica permite acceder al lenguaje de los mapas, pero también favorece el conocimiento del mundo en el que nos encontramos y la toma de decisiones (Castrogiovanni y Abreu, 2016). En consecuencia, la cartografía permite que el niño/a actúe y ejerza como un ciudadano crítico implicado en la construcción de un espacio geográfico común (Bittencourt y Kaercher, 2016).

\section{Marco empírico}

El estudio de casos realizado en segundo curso de EP tiene la finalidad de comprobar si los niños/as de esta etapa cuentan con una capacidad cartográfica mayor que la enunciada por la psicología evolutiva. Para ello se emplea un pretest y un postest validados en una prueba piloto (Jerez, 2014), con los que se analizan representaciones pictóricas y explicaciones escritas de los niños/as que permiten categorizar. De esta forma, se trata de una investigación de carácter cualitativo, ya que la propia percepción e interpretación de los niños/as sobre aspectos espaciales es clave para este estudio. Los métodos cualitativos permiten describir ciertos comportamientos y experiencias de las personas estudiadas y no tienen por qué ser menos rigurosos que los métodos cuantitativos (Taylor y Bogdan, 1992); además, el estudio de casos favorece la interpretación de las producciones y la comprensión de los resultados (Stake, 1999). 
Mapas personales y cartografía escolar: capacidades del alumnado de primer ciclo de educación primaria

María Jerez Carañana y Antonio José Morales Hernández

\section{Perfil de la muestra}

La muestra se compone por el alumnado de segundo curso perteneciente a un colegio situado en el Puerto de Sagunto (Valencia) durante el curso 2017-2018. El centro cuenta con dos líneas, por lo que existen dos grupos-clase: $2^{\circ} \mathrm{A}$, formado por 23 estudiantes; y $2^{\circ} \mathrm{B}$ con 21 alumnos/as (Tabla 1). Ambos grupos son bastante homogéneos y lo componen niños/ as de 7 y 8 años que viven en la localidad y que llevan una programación simultánea. La investigación tiene un diseño cuasiexperimental y predomina el análisis comparativo.

La variable se concreta en el proceso de alfabetización cartográfica, pues en $2^{\circ} \mathrm{A}$ se realiza una intervención con la finalidad de potenciar habilidades cartográficas en los niños/as. Con ello, el objeto de estudio está centrado en la posibilidad de que el grupo experimental evolucione en su aprendizaje cartográfico debido a la intervención realizada.

Tabla 1

Perfil de la muestra

\begin{tabular}{ccc}
\hline & Grupo experimental $\left(2^{\circ} \mathrm{A}\right)$ & Grupo control $\left(2^{\circ} \mathrm{B}\right)$ \\
\hline Pretest & 22 alumnos/as & 21 alumnos/as \\
Intervención & 23 alumnos/as* & - \\
Postest & 21 alumnos/as & 21 alumnos/as \\
\hline
\end{tabular}

Nota. *El alumnado del grupo experimental varía en número debido a que algunos estudiantes no acudieron a clase durante el pretest y el postest.

\section{Diseño de la experiencia}

El diseño de la experiencia está formado por la fase de diagnóstico, donde ambos grupos realizan un pretest; por la fase de intervención, aplicada sólo al grupo experimental durante seis sesiones de 45 minutos cada una y durante dos días a la semana; y por una fase de evaluación, llevada a cabo en ambos grupos a través de un postest (Tabla 2). Cabe decir que el postest se realizó un mes después de aplicar la sesión 6, pues se considera que ha pasado un tiempo suficiente para valorar el aprendizaje adquirido por los niños/as del grupo experimental. 
Tabla 2

Diseño de la experiencia

\begin{tabular}{|c|c|c|c|}
\hline Tipo de fase & Sesión & Grupo & Actividades realizadas \\
\hline Diagnóstica & Pretest & $\mathrm{A} / \mathrm{B}$ & Ficha con mapa mental sobre folio y preguntas. \\
\hline \multirow{6}{*}{ Intervención } & 1 & A & $\begin{array}{c}\text { Espacio vivido: nodos, mojones y descripción } \\
\text { de lugares en perspectiva horizontal. }\end{array}$ \\
\hline & 2 & A & $\begin{array}{l}\text { Espacio percibido: comparación de espacios } \\
\text { con perspectiva horizontal. }\end{array}$ \\
\hline & 3 & A & $\begin{array}{c}\text { Comparación de espacios con perspectiva hori- } \\
\text { zontal y oblicua. }\end{array}$ \\
\hline & 4 & A & Introducción a la perspectiva vertical. \\
\hline & 5 & A & Simbología \\
\hline & 6 & A & Detección de problemáticas de la localidad. \\
\hline Evaluación & Postest & $\mathrm{A} / \mathrm{B}$ & Ficha con mapa mental sobre plano y preguntas. \\
\hline
\end{tabular}

Con la intervención se ha aumentado en la complejidad del espacio observado: se parte del espacio vivido, con el reconocimiento y descripción de lugares próximos al alumnado, para pasar al espacio percibido y geométrico, donde predomina la perspectiva horizontal; después, dicha perspectiva se ha combinado con la oblicua, donde el alumnado ha podido establecer comparativas a partir de los espacios observados; y finalmente se ha iniciado al alumnado en el espacio absoluto, donde se ha trabajado la representación de objetos desde varias perspectivas, la localización, la interpretación de un plano y el establecimiento de símbolos.

En esta investigación tienen importancia los datos recogidos durante el pretest en ambos grupos, ya que la comparativa entre ambos permite comprobar si $2^{\circ} \mathrm{A}$ presenta un mayor control de las habilidades cartográficas que $2^{\circ} \mathrm{B}$. Para la categorización de los datos se han tomado como referencia autores destacados en este ámbito; todos ellos aluden al espacio subjetivo y a cómo el observador estructura y representa el espacio, por lo tanto, ofrecen las claves para analizar el contenido de las producciones del alumnado. Concretamente, Lynch (1960) expone que el observador estructura mentalmente el espacio en una serie de elementos, los cuales podrán reconocerse tanto en los mapas mentales como en la verbalización. Boardman (1986) argumenta y concreta los elementos cartográficos que los niños/as de EP son capaces de entender y utilizar con una instrucción adecuada, al igual que Bale (1989), quien añade a dichos elementos el trabajo con la perspectiva y la información adicional de los mapas (Tabla 3). 
Mapas personales y cartografía escolar: capacidades del alumnado de primer ciclo de educación primaria

María Jerez Carañana y Antonio José Morales Hernández

Tabla 3

Categorías empleadas para analizar las representaciones pictóricas

\begin{tabular}{lll}
\hline Lynch (1960) & Boardman (1986) & Bale (1989) \\
\hline C1. Sendas & C5. Dirección & C9. Perspectiva horizontal \\
C2. Nodos & C6. Localización & C10. Perspectiva oblicua \\
C3. Mojones & C7. Escala & C11. Perspectiva vertical \\
C4. Bordes & C8. Simbología & C12. Nombres \\
\hline
\end{tabular}

\section{Resultados y discusión}

En el pretest llevado a cabo durante la fase de diagnóstico se propone, en primer lugar, que el alumnado dibuje un mapa mental con la ruta casacolegio; y después, deben explicar por escrito el camino dibujado para valorar el dominio del código verbal. A través de estas producciones se extraen las categorías y se comprueba que los niños/as ya integran elementos cartográficos sin ninguna instrucción previa.

Por otro lado, en el postest de la fase de evaluación, se ofrece al alumnado un plano a gran escala en tamaño A3 donde deben marcar el itinerario que seguirían para ir desde el colegio a la playa, así como la explicación escrita. Esta propuesta es más compleja porque hay una perspectiva vertical y es una ruta menos familiar para ellos/as, lo que permite comprobar si el grupo experimental tiene una mayor capacidad cartográfica al integrar más elementos.

Casi la totalidad del alumnado representó en sus dibujos un camino, o bien una línea continua o discontinua. Lynch (1960) lo denomina como la senda, que es el camino que sigue el observador; por lo que este elemento se corresponde con la categoría 1. Además, se observa que el alumnado marcaba un punto de origen y otro de destino, y se conectaban mediante la senda. El autor los define como nodos, pues son puntos en los que el observador se introduce o de los que parte, y ha dado lugar a la categoría 2. Los niños/as han representado los nodos dibujando la casa propia, el colegio y la playa, o bien indicándolo por escrito.

Los mojones son la categoría 3, descritos por Lynch (1960) como aquellos lugares que sirven de referencia al observador durante el recorrido sin llegar a introducirse en ellos. Los niños/as los asocian con lugares significativos para ellos/as y suelen ser espacios públicos, espacios naturales, elementos viales y comercios. En el mapa mental del pretest, los mojones aparecen a través de un dibujo o de forma escrita (Figura 
1); mientras que en el postest, muchos de los niños/as del grupo experimental abstraen lo mojones empleando símbolos o formas geométricas, como cuadrados o rectángulos.

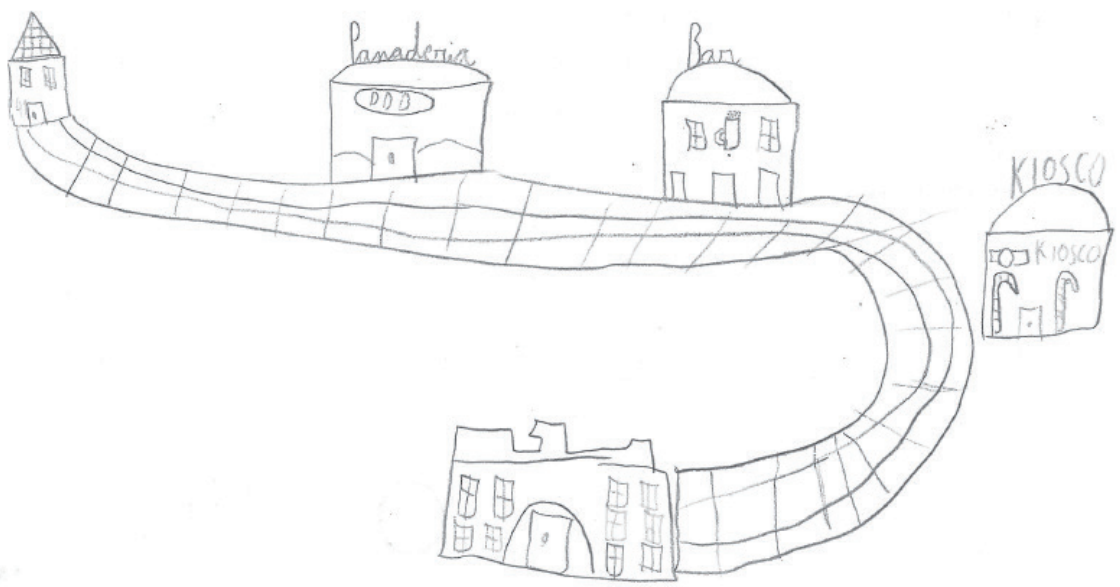

Figura 1. Ejemplo de mapa mental con mojones.

Fuente: alumno $\mathrm{n}^{\circ} 6$ de $2^{\circ} \mathrm{A}(2018)$

El último elemento establecido por Lynch (1960) tiene que ver con los bordes, empleados como categoría 4. Aunque han aparecido menos, sí hay niños/as que los han asociado con límites o fronteras naturales, ya que dibujaron el mar o la desembocadura del río Palancia, ambos muy próximos al colegio.

Las siguientes categorías son proporcionadas por Boardman (1986), quien establece los elementos cartográficos esenciales que son capaces de comprender los niños/as de EP. En los dibujos, los niños/as han empleado flechas para indicar la dirección, que se ha establecido como categoría 5. Se entiende como la ruta que los niños/as planifican para ir de un sitio a otro. Verbalmente la dirección se corresponde con el movimiento que debe hacer la persona para seguir la ruta, pues los niños/as emplean palabras como "girar a la derecha" o "recto". 


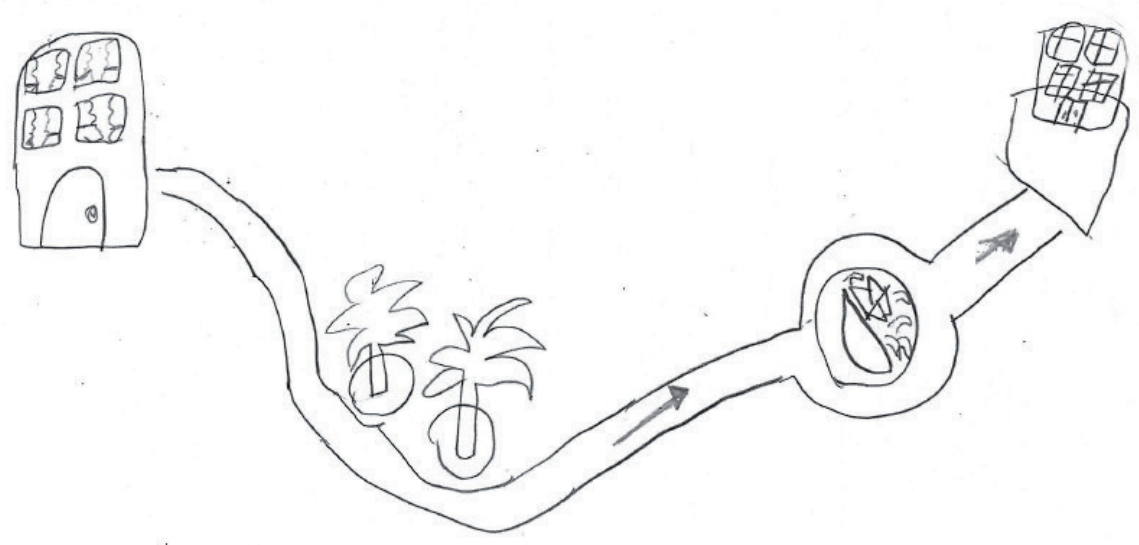

Figura 2. Ejemplo de mapa mental con dirección.

Fuente: alumno $\mathrm{n}^{\mathrm{O}} 12$ de $2^{\circ} \mathrm{A}(2018)$

La categoría 6 se corresponde con la localización, que es empleada por los niños/as para representar una posición concreta de un lugar en el espacio y resaltar su importancia. Tras el análisis de las representaciones pictóricas se ha observado que los niños/as localizan espacios similares, como el parque, la casa propia y el colegio. Pictóricamente, la muestra localiza con rectángulos, cuadrados o círculos que incluyen dentro el topónimo o un dibujo de ese lugar, o bien que usan equis y puntos. Verbalmente, los niños/as localizan cuando toman de referencia lugares externos a sí mismos; por ejemplo emplean palabras como "derecha", "al lado" o "detrás".

La siguiente categoría tiene que ver con la escala, que en el primer ciclo de EP se representa a través de la proporcionalidad (Piñeiro, 1998). En el mapa mental, los niños/as representan el espacio a gran escala y lo reducen a una sola calle o camino; además las proporciones se distorsionan al representar los nodos, pues la casa propia y el colegio se dibujan más grandes que el resto de lugares. Verbalmente, la escala se asocia con el tamaño (Marrón, 2012) y emplean palabras como "grande" o "pequeño".

Boardman (1986) también argumenta que los niños/as de EP pueden llegar a ser capaces de incluir símbolos en mapas y por ello se ha seleccionado como categoría 8 . A través de las representaciones pictóricas se observa que la muestra incluye símbolos convencionales pero también inventados. En el pretest, los símbolos acompañaban al dibujo del lugar o al nombre escrito en el mapa mental; mientras que en postest, el gru- 
po experimental demostró un mayor dominio de los mismos al emplear únicamente el dibujo y omitir los nombres escritos al lado.

El tercer autor que ha servido de referencia para la categorización de los elementos cartográficos es Bale (1989). En su obra hace alusión a la importancia del trabajo de la perspectiva, que se ha dividido en las siguientes categorías: la número 9 o perspectiva horizontal, donde los niños/as dibujan los elementos en el mapa vistos a nivel del suelo; la 10 o perspectiva oblicua, donde los elementos se representan con mayor altura; y la número 11 o perspectiva vertical, donde la visión del espacio es desde arriba. En el pretest la visión predominante es la horizontal, pues los niños/as tienden a representar el espacio tal y como lo observan diariamente. Sin embargo, se ha comprobado que en segundo curso algunos niños/as ya cuentan con una serie de capacidades cartográficas y que son capaces de abstraer el espacio al representarlo en perspectiva vertical sin instrucción previa (Figura 3).

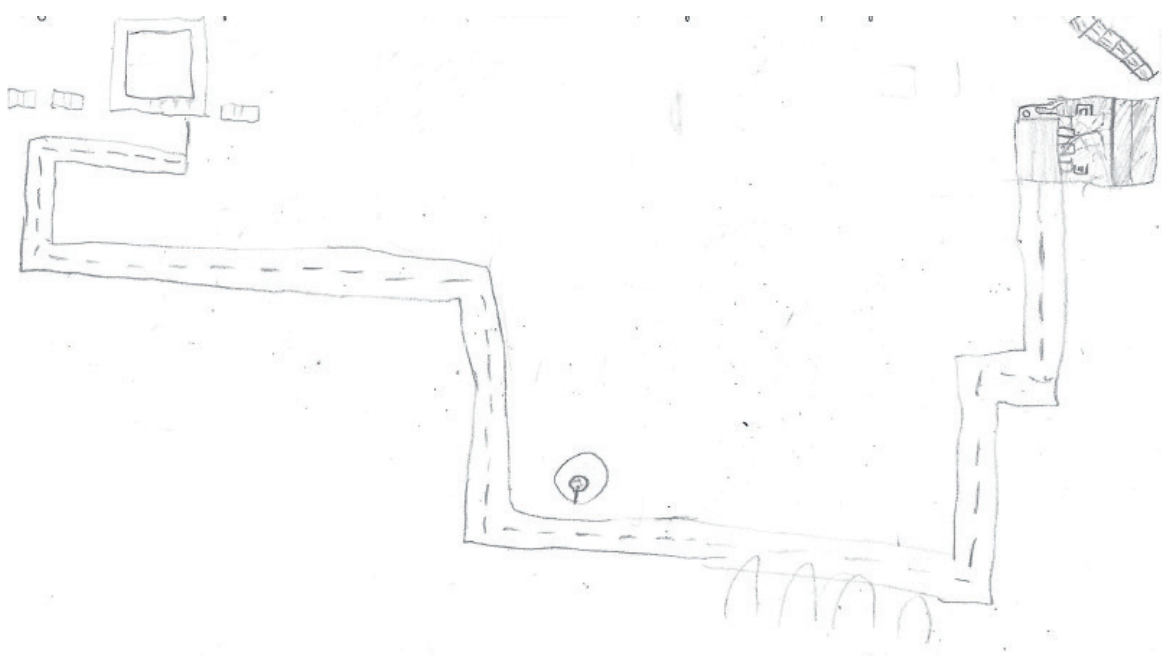

Figura 3. Ejemplo de mapa mental con perspectiva vertical. Fuente: alumno $\mathrm{n}^{\circ} 18$ de $2^{\circ} \mathrm{A}(2018)$

Por último, Bale (1989) argumenta que los mapas incluyen información adicional y se asocia con los nombres, lo que da lugar a la categoría 12. La muestra los emplea en las representaciones pictóricas para clarificar el lugar que se ha dibujado o localizado, y también destacan en las explicaciones verbales del recorrido. 
Tanto el pretest como el postest se componen de otra propuesta que permite recoger información sobre la capacidad de percepción del espacio mediante las TIC. Concretamente, en el pretest, los niños/as deben explicar por escrito cómo ven el colegio con la aplicación Google Earth; mientras que en el postest deben localizar la playa sobre el plano y reflexionar sobre la utilidad de ver el espacio en perspectiva vertical.

A partir de las respuestas del alumnado surgen categorías que no estaban previstas, de manera que las explicaciones verbales sirven para aportar nueva información sobre la forma en la que el niño/a percibe el espacio, así como complementar la información ofrecida por los mapas mentales. Aquí aparece la categoría 13 sobre la comprensión. Los niños/ as de estas edades son conscientes de que la perspectiva vertical que posee el mapa facilita la comprensión y el conocimiento del espacio, aspecto que según Hernández (2002) también debe caracterizar a la didáctica de las Ciencias Sociales. Además, se comprueba que los niños/ as son conscientes de que los mapas omiten elementos para abstraer el espacio y el contenido de los mismos varía según la escala, idea que también contempla la propia LOMCE; de forma que la categoría 14 se asocia con el contenido. Por último, la categoría 15 tiene que ver con la forma, pues tal y como expone Souto (1998) los niños/as de estas edades tienen la capacidad de discriminar figuras geométricas en los planos, aspecto que se ha comprobado al describir el colegio con Google Earth.

Tras la exposición de las categorías, quedan sintetizados los datos recogidos durante el pretest y el postest (Tabla 4).

Tabla 4

Conjunto de los elementos cartográficos presentes en las representaciones pictóricas

\begin{tabular}{cccccc}
\hline & & \multicolumn{2}{c}{ Pretest } & \multicolumn{2}{c}{ Postest } \\
\cline { 3 - 6 } & & \multicolumn{1}{c}{ Control } & Experimental* & Control & Experimental \\
\cline { 3 - 6 } & & $2^{\circ} \mathrm{B}$ & $2^{\circ} \mathrm{A}$ & $2^{\circ} \mathrm{B}$ & $2^{\circ} \mathrm{A}$ \\
\hline \multirow{3}{*}{ Lynch } & C1.Sendas & 19 & 20 & 21 & 21 \\
$(1960)$ & C2. Nodos & 21 & 22 & 21 & 21 \\
& C3. Mojones & 16 & 20 & 14 & 21 \\
\hline \multirow{2}{*}{ Boardman } & C5. Bordes & 1 & 1 & - & - \\
$(1986)$ & C6. Dirección & 20 & 20 & 21 & 21 \\
& C7. Escalización & 5 & 4 & 6 & 16 \\
& C8. Simbología & 14 & 11 & 3 & 10 \\
\hline
\end{tabular}




\begin{tabular}{cccccc}
\hline & $\begin{array}{c}\text { C9. Perspectiva } \\
\text { horizontal }\end{array}$ & 21 & 21 & 9 & 5 \\
Bale & $\begin{array}{c}\text { C10. Perspectiva } \\
\text { oblicua }\end{array}$ & 3 & 2 & - & - \\
(1989) & $\begin{array}{c}\text { C11. Perspectiva } \\
\text { vertical }\end{array}$ & 4 & 8 & 4 & 13 \\
& C12. Nombres & 14 & 13 & 10 & 8 \\
\hline
\end{tabular}

Nota.*El grupo control cuenta con 21 alumnos/as, mientras que el grupo experimental tiene 22 en el pretest y 21 en el postest debido a que algunos estudiantes no acudieron a clase en la última sesión.

Mediante la tabla se contempla que las sendas, nodos, mojones y bordes estructuran la imagen mental que tiene el observador sobre su entorno, pues los niños/as los representan desde el principio. En cambio el grupo experimental representa más mojones y usa los lugares conocidos para guiarse en el recorrido del postest, demostrando mayor capacidad para reconocer espacios y para emplear la memoria episódica, relacionada con los recuerdos de las propias experiencias. Esta capacidad puede haberse consolidado durante la intervención en $2^{\circ} \mathrm{A}$, pues se incidió en la descripción y el reconocimiento de lugares para trabajar desde los espacios vividos y percibidos, es decir, aquellos que forman parte del espacio subjetivo y de la cartografía personal. De esta forma, los niños de $2^{\circ}$ curso son capaces de recurrir al espacio subjetivo para reconocer lugares en el plano e integrarlos en el espacio absoluto.

En la fase de diagnóstico se ha observado que los niños/as agrupan todos los elementos cerca de la senda y que el espacio representado es a gran escala y limitado, por lo que se debe ir trabajando la distribución de estos elementos para permitir al lector orientarse y ubicarse a través de la imagen.

El trazado pictórico predomina en el pretest, pero sobre el plano del postest los niños/as tratan de simplificar el espacio y predomina un trazado más esquemático. De esta forma, se comprueba que los niños/as de estas edades cuentan con la capacidad para trazar bidimensionalmente elementos que son tridimensionales. Por lo tanto, en primer ciclo de EP sí es posible iniciar a los niños/as en representaciones proyectivas, contrariamente a la idea piagetiana de que en estas edades predomina la representación topológica y egocéntrica.

La escala es otro aspecto diferenciador en ambos grupos, pues el 
experimental la emplea mucho más sobre el plano. A pesar de esto, las proporciones todavía no están claras en la mitad del grupo. Según los postulados piagetianos, esta dificultad se debe a que el niño/a de primer ciclo de EP todavía no está preparado para representar adecuadamente las distancias, las direcciones o las dimensiones. Con esta investigación se ha comprobado que dicha dificultad existe pero que puede solventarse, ya que los resultados obtenidos demuestran que es posible iniciar a los niños/as de primer ciclo de EP en la observación y representación de espacios de forma competente, así como en la localización de nodos o mojones, y en la distribución y ordenación de éstos en el espacio; impugnando aquello que está establecido por la psicología evolutiva.

Respecto a la localización y los símbolos, se observa que $2^{\circ} \mathrm{A}$ también lo ha empleado adecuadamente sobre el plano. En el pretest, los símbolos acompañan a nombres o a dibujos de los lugares, pero ahora los niños/as ya han comprendido sólo el símbolo ya es suficiente para representar ciertos lugares. Además, han resaltado su importancia localizándolos e insertándolos en cuadrados o rectángulos. Esto, a su vez, favorece el uso de la perspectiva vertical, cuyo trazo es asociado por los niños/as con el contorno. Con todos estos progresos se comprueba que la intervención ha sido beneficiosa para el grupo experimental y que los niños/as se han iniciado en la adquisición de la alfabetización cartográfica.

Por último, a diferencia de los expuesto por la LOMCE, se considera que el aprendizaje de la cartografía se debe hacer de forma global e interdisciplinar, pues se ha demostrado que las destrezas que requiere la cartografía están relacionadas y no deberían aprenderse de forma aislada o fragmentada en cursos, tal y como establece el currículo de EP.

\section{Conclusiones}

Tras consultar la secuencia de los contenidos cartográficos y los criterios de evaluación establecidos por la LOMCE, se comprueba que la cartografía escolar está regida por la lógica del desarrollo cognitivo, dando por hecho que los niños/as de EP todavía no tienen la capacidad de abstracción necesaria para realizar representaciones espaciales en perspectiva vertical o para incluir por sí mismos elementos cartográficos. Por 
el contrario, con el análisis de las representaciones pictóricas y los datos recogidos durante el pretest, se comprueba que los escolares de 7-8 años ya tienen la capacidad de abstraer el espacio y estructurarlo, así como de incluir elementos cartográficos o de emplear la perspectiva vertical; todo ello de forma intuitiva y sin un entrenamiento previo. Cabe decir que se ha observado que el alumnado de esta etapa presenta limitaciones a la hora de comprender o representar el código cartográfico, pero también ha quedado demostrado a través de los resultados del grupo experimental que las capacidades cartográficas pueden mejorarse si se ejercitan mediante una instrucción adecuada. Esta idea ya había sido defendida por autores expertos en la materia, tanto a nivel nacional (Comes, 1993; Souto 1998) como internacional (Lynch, 1960; Boardman, 1986; Bale, 1989), quienes establecían como elementos básicos en el aprendizaje cartográfico la existencia de una secuenciación de tareas en base a la capacidad cartográfica del niño/a así como la importancia de que se vincule el espacio subjetivo y personal con el absoluto y objetivo. Este aspecto se ha comprobado a través del postest, pues se ha evidenciado que para la enseñanza y el aprendizaje de la cartografía es beneficioso recurrir al espacio subjetivo y que éste facilita a los niños/as la comprensión del espacio absoluto. De esta forma, con la fase de evaluación, se corrobora que las habilidades cartográficas pueden ir asentándose a través de la práctica y de la experiencia, tal y como ya señalaban los postulados socioconstructivistas.

Por otro lado, con la diagnosis de la cartografía personal, se observa que el alumnado de segundo curso de EP tiene interiorizados los elementos que sirven para estructurar la imagen mental del espacio según Lynch (1960), ya que las sendas, nodos, mojones y bordes han sido representados en los dibujos iniciales. El espacio representado es limitado y se compone casi exclusivamente de nodos y mojones que quedan agrupados cerca de la senda; es decir, en su representación espacial obvian espacios poco conocidos y priorizan los más significativos, que los asocian con lugares de consumo y ocio o de encuentro con personas conocidas. Éstos forman parte de su propia identidad y configuran su imagen espacial. Además, los resultados del postest indican que la competencia cartográfica se asume mejor si se parte de los espacios significativos de la infancia, pues el grupo experimental mejora en la lectura del plano, en el reconocimiento de lugares y en la representación de los mismos. Por tanto, se comprueba que el alumnado recurre al espacio vi- 
vido y percibido para llevar a cabo dichas habilidades y que es capaz de integrar estos espacios en el espacio absoluto, potenciado así la competencia cartográfica. Todo esto lleva al pensamiento de que la cartografía personal debería ser imprescindible en el ámbito educativo, ya que hay aspectos subjetivos, biológicos y sociales que deben ser tenidos en cuenta en las escuelas con la finalidad de alcanzar las destrezas cartográficas; y todo ello desde una perspectiva socioconstructivista, donde se prioriza la experiencia.

Siguiendo con los elementos empleados en las representaciones pictóricas y mapas mentales, se puede recabar más información sobre la capacidad que tienen los niños/as de $2^{\circ}$ de EP sobre la capacidad de abstracción del espacio. A través de la categoría de escala se comprueba la geometría euclidiana no está asentada a los 7 y 8 años, es decir, las dimensiones y las distancias mostradas por los niños/as no se adecuan a la realidad; aunque sí se observa la capacidad de representar el espacio de forma proyectiva, ya que inicialmente empleaban más la perspectiva horizontal y los iconos y, tras la intervención, hay un mayor uso de la perspectiva vertical y de los elementos en dos dimensiones. Además, se ha evidenciado que los los escolares de $2^{\circ}$ curso de Educación Primaria son capaces de emplear símbolos e integrarlos en la representación cartográfica.

Por otro lado, el análisis sobre el código verbal ha servido para aportar información a la ya recogida en los mapas mentales e itinerarios, hasta el punto de que se han contemplado tres nuevas categorías no previstas inicialmente. Por tanto, el lenguaje escrito ayuda a comprender y valorar mejor el nivel de competencia cartográfica que posee el alumnado.

Por último, se ha evidenciado que la aplicación de una secuencia de actividades en el grupo experimental ha derivado en que los niños/ as mejoren en su competencia cartográfica, ya que su manejo de los mojones, localización, escala, simbología y perspectiva vertical ha aumentado considerablemente en comparación con el grupo control. Todo esto lleva a corroborar la hipótesis de partida, mediante indicios que evidencian que la competencia cartográfica no se adquiere tan solo por madurez, sino que está condicionada por la experiencia, motivo por el cual los resultados de este estudio de casos también podrían ser extensibles a primero de EP. De esta forma, el alumnado de primer ciclo de EP poseería mayores capacidades para la cartografía que aquellas que 
describe la psicología evolutiva y es posible una mejora de las mismas, siempre y cuando se parta de sus cartografías personales y se establezcan relaciones entre el espacio subjetivo y el absoluto.

Las limitaciones de esta investigación han sido las propias de un estudio de casos, en relación a que se ha tratado con una muestra reducida y los datos deben considerarse como indicios. Las líneas de investigación futuras, aplicadas a un mayor número de alumnado y otros ciclos de la Educación Primaria, nos pueden ayudar a consolidar los datos obtenidos y con ello mejorar los criterios de evaluación recogidos en la legislación vigente respecto al aprendizaje de la cartografía escolar.

\section{Referencias}

Bale, J. (1989). Didáctica de la geografía en la escuela primaria. Madrid: Ediciones Morata.

Bittencourt, J. y Kaercher, N. (2016). Os arredores da escola: lugarizando a aprendizagem, vivenciando a geografia por meio de maquetes e cordel. En A. Castrogiovanni, I. Tonini, N. Kaercher, y R. Costella (Orgs.). Movimentos para ensinar Geografia - oscilações (p. 255-277). Porto Alegre: Editora Letra 1. http://dx.doi.org/10.21826/9788 $563800244 \mathrm{p} 255-277$

Boira, J. V., Reques, P. y Souto, X. M. (1994). Espacio subjetivo y geografía. Orientación teórica y praxis didáctica. Valencia: NAU Ilibres.

Boardman, D. (1986). Maps and Mapwork. En D. Boardman, D. Carter y H. Sandford, Handbook for geography teachers (p. 123-148). Sheffield: Geographical Association.

Castrogiovanni, A. y Abreu, P. R. (2016). A dialógica entre a Cartografia no ensino básico e o sistema de informação geográfica nos pleitos territoriais. En A. Castrogiovanni, I. Tonini, N. Kaercher, y R. Costella (Orgs.). Movimentos para ensinar Geografia - oscilações (p.143-156). Porto Alegre: Editora Letra 1. http://dx.doi.org/10.21826/97885 63800244p143-156

Cavalcanti, L. (2017). El análisis de la espacialidad y la comprensión del mundo: Ilave para la relevancia de la geografía escolar. En R. Sebastiá y E. Tonda. (Coords.), Enseñanza y aprendizaje de la geografía para el siglo XXI (p. 97-116). Alicante: Universidad de Alicante.

Comes, P. (1993). Los procedimientos en geografía. Una propuesta de clasificación y secuenciación de las habilidades cartográficas en la enseñanza obligatoria (6-16 años). AULA de Innovación Educativa, 10, 28-33.

Decreto 108/2014 de 4 de julio, del Consell, por el que se establece el currículo y desarrolla la ordenación general de la Educación Primaria en la Comunitat Valenciana.

Francischett, M. (2016). A didáctica na cartografia escolar no ensino fundamental de geografía. En S. Alcaraz y E. Tonda (Eds.). La investigación e innovación en la ense- 
Mapas personales y cartografía escolar: capacidades del alumnado de primer ciclo de educación primaria

María Jerez Carañana y Antonio José Morales Hernández

ñanza de la Geografía (p. 353-362). San Vicente del Raspeig: Universidad de Alicante. http://dx.doi.org/10.14198/GeoAlicante2015.25

Gardner, H. (2003). Inteligencias múltiples. La teoría en la práctica. Barcelona: Paidós.

Hannoun, H. (1977). El niño conquista el medio. Buenos aires: Kapelusz.

Hernández, F. X. (2002). Didáctica de las Ciencias Sociales, geografía e historia. Barcelona: Graó.

Jerez. (2014). Construyendo el espacio geográfico a partir del mundo subjetivo de los alumnos. (Trabajo de Fin de Grado). Universitat de València, Valencia. Recuperado de http://roderic.uv.es/handle/10550/43946

Jerez. (2016). Aprender el espacio donde vivimos. AULA de Innovación Educativa, 248, 33-38.

Jiménez, M., Martínez, M., Castillo, F. y López, R. (2019). Miradas en los espejos: reflexiones sobre cómo aprendemos ciencias y cómo la enseñamos. En M. Ferreras, O. Moreno y M. Fernández (Coords.), Innovación e investigación en la formación inicial del profesorado de Infantil y Primaria desde las Didácticas de las Ciencias Experimentales y Sociales (p. 155-176). Barcelona: Ediciones Octaedro.

Ley Orgánica 8/2013, de 9 diciembre, para la mejora de la calidad educativa.

Lynch, K. (1960). La imagen de la ciudad. Barcelona: Gustavo Gili.

Marrón, M. J. (2012). Aproximación al concepto de escala en el primer ciclo de Educación Primaria. Presentación de un juego para su tratamiento desde la enseñanza activa. Didáctica Geográfica, 13, 93-112. Recuperado de https://didacticageografica. age-geografia.es/index.php/didacticageografica/article/view/98

Martínez, R. y López, J. (2015). Análisis de la cartografía en los libros de texto de Ciencias Sociales en Educación Primaria. En J. De la Riva, P. Ibarra, R. Montorio, M. Rodrigues (Eds.). Análisis espacial y representación geográfica: innovación y aplicación (p.1455-1464). Universidad de Zaragoza-AGE. Recuperado de http://congresoage. unizar.es/eBook/trabajos/153_Martinez\%20Medina.pdf

Ortiz, A., Prats, M. y Baylina, M. (2011). Métodos visuales y geografías de la infancia: dibujando el entorno cotidiano. Scripta Nova, Revista Electrónica de Geografía y Ciencias Sociales. XVI (400). Recuperado de http://www.ub.edu/geocrit/sn/sn-400. htm

Piaget, J. e Inhelder, B. (1947). La représentatition de l'espace chez l'infant. Paris: PUF.

Piaget, J. e Inhelder, B. (2015). Psicología del niño (Juan Delval, trad.). Morata: Madrid. (Obra original publicada en 1969). Recuperado de https://issuu.com/ediciones_morata/docs/piaget_psicolog_a_del_ni_o

Pillet, F. (2004). La geografía y las distintas acepciones del espacio geográfico. Investigaciones geográficas, 34, 141-154.

Piñeiro, M ${ }^{a}$. R. (1998). Dirección y Orientación en Educación Infantil y Primaria. En P. Pérez, Ma . R. Piñeiro y C. Tirado, Enseñar y aprender el espacio geográfico. Un proyecto de trabajo para la comprensión inicial del espacio: Orientación teórica y praxis didáctica (p. 5-24). Valencia: Nau Llibres.

Souto, X. M. (1998). Didáctica de la Geografía. Problemas sociales y conocimiento del medio. Barcelona: Ediciones del Serbal.

Souto, X. M. (2011). Didáctica de la Geografía y otras ciencias sociales. En J. Prats, R. 
Mapas personales y cartografía escolar: capacidades del alumnado de primer ciclo de educación primaria María Jerez Carañana y Antonio José Morales Hernández

Prieto-Puga, J. Santacana, X. M. Souto y C. Trepat, Didáctica de la geografía y la historia (p. 113-171). Barcelona: Graó.

Stake, R. E. (1999). Investigación con estudio de casos. Madrid: Ediciones Morata.

Taylor, S. y Bogdan, R. (1992). Introducción a los Métodos Cualitativos de Investigación. Buenos Aires: Paidós Básica.

Tonda, E. (2001). La Didáctica de las Ciencias Sociales en la formación del profesorado de Educación Infantil. Alicante: Publicaciones Universidad de Alicante.

Thurstone, L. (1938). Primary mental abilities. Chicago: University of Chicago Press.

Vygotsky, L. (1962). Thought and Languaje. Cambridge: The MLT Press. 
